\title{
Colonoscopic finding of an unusual sigmoid colon fistula caused by ovarian teratoma
}

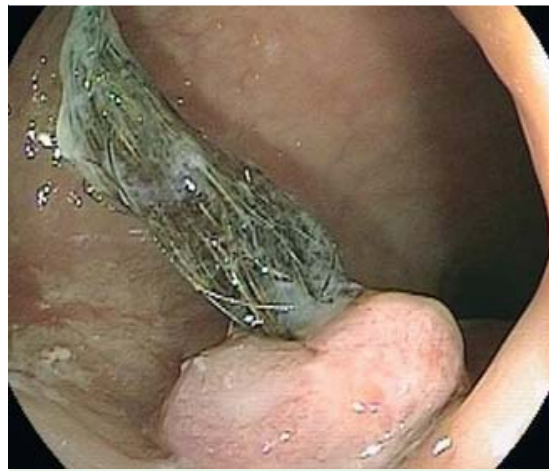

Fig. 1 Colonoscopic view of the fistula in the sigmoid colon and hair strands protruding into the lumen.

A 42-year-old woman was admitted to our hospital because of intermittent lower abdominal pain with mild constipation over the preceding 4 months. Her medical history was uneventful and we recommended that she undergo a colonoscopy. During the procedure, we discovered to our surprise a fistula in the colon $(16 \mathrm{~cm}$ from the anal verge near the rectosigmoid junction) with swollen surrounding mucosa and a tuft of hair that was protruding straight into the colonic lumen ( $\bullet$ Fig. 1; - Video 1). Our endoscopic diagnosis was of a fistula of the sigmoid colon with the suspicion of an underlying teratoma. An abdominal computed tomography (CT) scan revealed a heterogeneous left ovarian mass in close contact with the sigmoid colon ( Fig. 2).

The patient was subsequently referred for surgery. During the course of this, a large mass that was arising from the left ovary and protruding into the colonic lumen was identified. The perforation that this was causing was clearly visible in the anterior wall of the sigmoid colon ( $\bullet$ Fig.3). A left oophorectomy with en bloc excision of the teratoma was performed, and the involved sigmoid colon was resected, with a colorectal anastomosis being created. The patient had no complications and was discharged 1 week after her surgery.

\section{Video 1}

A fistula of the sigmoid colon with hair strands protruding into the lumen that was unexpectedly found at colonoscopy.

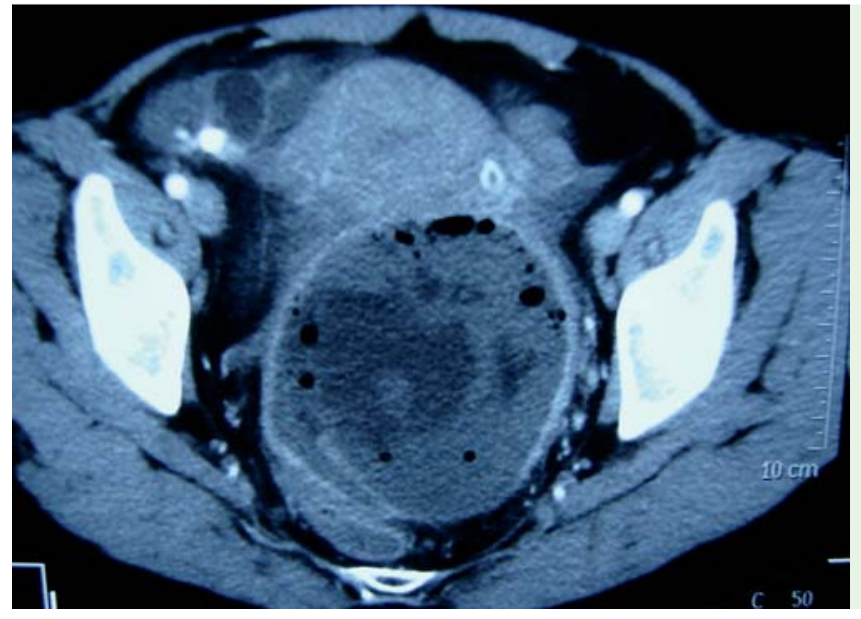

Fig. 2 Pelvic computed tomography (CT) scan showing an $11 \mathrm{~cm} \times 11 \mathrm{~cm}$ heterogeneous ovarian mass compressing the colon.

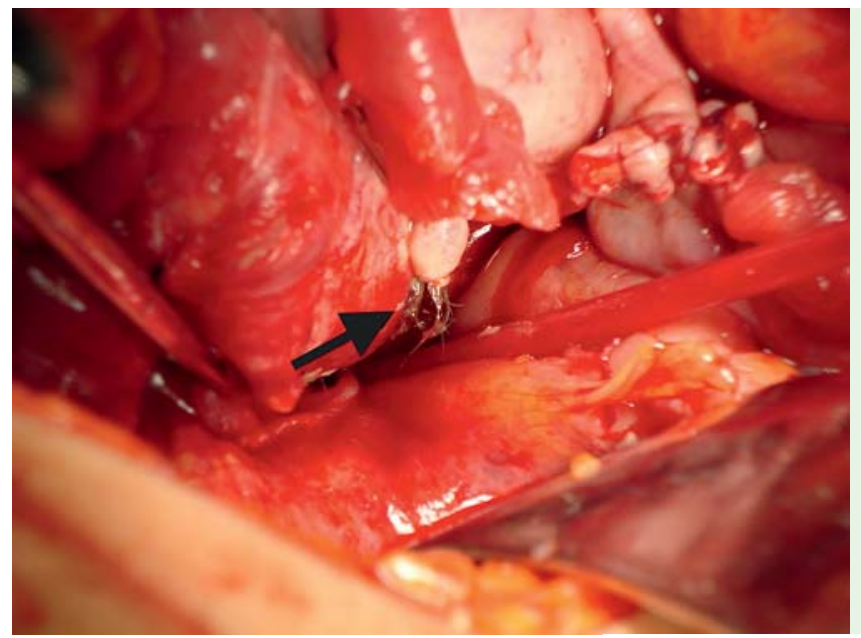

Fig. 3 Surgical view showing the left ovarian teratoma infiltrating the colon (arrow).

The resected tumor specimen was $11 \mathrm{~cm} \times$ $11 \mathrm{~cm}$ in size and consisted of hairs and smelly fluid ( Fig.4). Pathological examination revealed a mature cystic ovarian teratoma that had ruptured and infiltrated into the lumen of the sigmoid colon producing the fistula.

Colonic fistulas caused by infiltrating teratomas, which can originate from the ovary or even from the colon itself, are extremely rare $[1,2]$. Complications of ovarian teratoma include infection, torsion, rupture, and malignant transformation [2]. Rarely, the tumor can rupture into the pelvic cavity or adjacent organs, such as the colon and bladder, causing corresponding symptoms [3]. This case represents a rare complication, with a ruptured ovarian teratoma presenting as a colonic fistula that was initially discovered on screening colonoscopy. Surgery may be helpful in treating these patients.

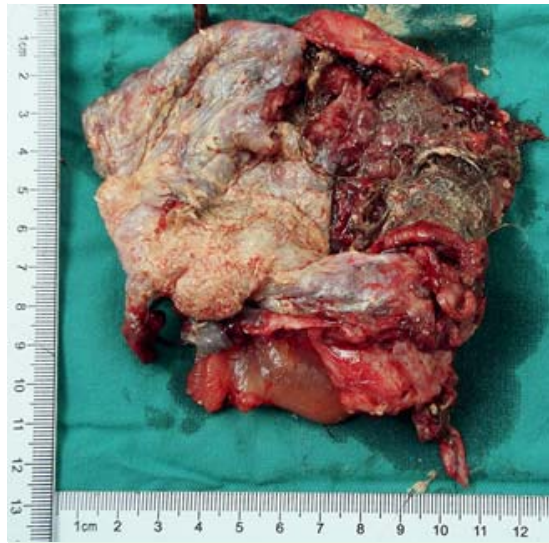

Fig. 4 Macroscopic view of the opened surgical specimen showing the presence of hairs in the ovarian teratoma. 
Endoscopy_UCTN_Code_CCL_1AD_2AG

Competing interests: None

Hang Yi, Yi Mou, Wei Liu, Hongze Zeng, Qiming Wang, Chengwei Tang, Bing $\mathrm{Hu}$

Digestive Endoscopy Center, Department of Gastroenterology, West China Hospital, Sichuan University, Chengdu, Sichuan,

China

\section{References}

1 Salame G, Sherer DM, Shah T et al. Mature cystic teratoma of the sigmoid colon. Ultrasound Obstet Gynecol 2011; 37: 739-740

2 Park HC, Park SH, Kim W et al. Ovarian teratoma presenting as a pedunculated polyp at colonoscopy. Endoscopy 2006; 38 (Suppl. 02): E36

3 von-Walter AR, Nelken RS. Benign cystic ovarian teratoma with a fistula into the small and large bowel. Obstet Gynecol 2012; 119: 434-436
Bibliography

Dol http://dx.doi.org/

10.1055/s-0034-1391236

Endoscopy 2015; 47: E85-E86

(c) Georg Thieme Verlag KC

Stuttgart · New York

ISSN 0013-726X

\section{Corresponding author}

Bing Hu, MD

Digestive Endoscopy Center

Department of Gastroenterology

West China Hospital

Sichuan University

Chengdu

Sichuan, 610041

China

hubingnj@163.com 